\title{
Um processo de elicitação de requisitos com foco na seleção da técnica de elicitação
}

\author{
Glívia Barbosa ${ }^{1}$, Marcelo Werneck ${ }^{1}$, Helen Assis ${ }^{1}$, Ulisses Fernandes ${ }^{1}$, Ismael \\ Silva ${ }^{1}$ \\ ${ }^{1}$ Instituto de Informática - Pontifícia Universidade Católica de Minas Gerais (PUC \\ Minas) - Belo Horizonte - MG - Brasil \\ \{mwerneck\} @pucminas.br, \{gliviaangelica, hfbassis, ufernandes, \\ ismasantana\} @gmail. com
}

Resumo. Este trabalho apresenta um processo focado na seleção da técnica de elicitação de requisitos. Na tentativa de garantir que os requisitos relevantes para o sistema sejam elicitados de forma correta e completa, um conjunto de técnicas de elicitação pode ser aplicado com o intuito de auxiliar os analistas e usuários na identificação e definição desses requisitos. Porém, a elicitação não se resume apenas à aplicação de uma técnica, mas também à colaboração entre analistas e clientes. Este trabalho apresenta um processo para elicitação de requisitos com foco na escolha da técnica de elicitação para o projeto em questão contemplando atividades e artefatos que visam maior colaboração entre os envolvidos.

Abstract. This work presents a process focused on the selection of the requirements elicitation technique. In an attempt to ensure that the relevant system requirements are correctly and completely elicited, a set of techniques can be applied aiming at helping the analysts and users identify and define these requirements. However, the elicitation is not simply the application of a technique but also the cooperation between analysts and clients. This paper presents a requirements elicitation process that focuses on aiding analysts selecting the best elicitation technique to be used in that particular project. The process also includes activities and artifacts to achieve greater collaboration among those involved in the elicitation.

\section{Introdução}

A Engenharia de Requisitos contempla um conjunto de atividades relacionadas à definição e gerência dos requisitos. Dentre essas atividades, podemos destacar: elicitar, analisar, documentar, validar estes requisitos e, durante todo o desenvolvimento de software, gerenciá-los [Pfleeger, 2004].

Uma etapa crítica da engenharia de requisitos é a elicitação, fase complexa da definição de requisitos e de todo processo de desenvolvimento de software, uma vez que é base para todas as etapas posteriores [Belgamo e Martins, 2000]. Elicitar requisitos requer um processo iterativo, que deve acontecer de forma colaborativa envolvendo analistas, usuários e aplicação de técnicas [Freitas, Borges e Araújo, 2007].

Um estudo realizado pelo Standish Group [Standish report, 1995], com 350 companhias e 8.000 projetos indica que 16,2\% dos projetos são concluídos com sucesso; $52,7 \%$ são considerados problemáticos, pois não atendem às necessidades dos usuários com custos excessivos e $31,1 \%$ são cancelados antes de serem completados. 
Buscando identificar quais fatores levam à distribuição apresentada, nova pesquisa foi realizada e 8 fatores foram identificados como críticos, dos quais 3 estão relacionados à elicitação e gerência dos requisitos. São eles: "Requisitos incompletos", "Falta de envolvimento do usuário" e "Mudanças de requisitos e especificações".

A forma como a elicitação de requisitos é feita influencia o produto desenvolvido. Os requisitos levantados são um indicador importante para o fracasso ou sucesso do projeto de um software. Requisitos mal levantados ou mal interpretados geram retrabalho, custos e prazos extras, além de insatisfação do cliente.

Existem algumas técnicas que buscam auxiliar os analistas e os usuários na identificação dos requisitos do sistema, contudo, a complexidade dessa etapa de elicitação se deve não apenas a fatores técnicos, mas também humanos.

As técnicas de elicitação auxiliam o desenvolvimento de software, mas os problemas essenciais da elicitação são um desafio que ainda precisa ser superado. Uma possível solução mesclada às técnicas e ferramentas que poderá resolver estes problemas essenciais é o uso de um processo que promova um trabalho colaborativo entre a equipe e os usuários certos, ou seja, aqueles que realmente contribuem para a identificação dos requisitos [Freitas, Borges e Araújo, 2007].

Escolher e aplicar uma técnica de elicitação não é uma tarefa trivial, porém reduzir a fase de elicitação à utilização de técnicas apenas não garante que os requisitos identificados satisfaçam o cliente final [Belgamo e Martins, 2000].

Algumas técnicas que facilitam a comunicação entre clientes e analistas têm sido usadas visando minimizar problemas encontrados na elicitação de requisitos. Tem sido observado, porém, que a forma com que estas técnicas têm sido aplicadas ainda não solucionou os problemas da elicitação de requisitos. Constata-se que, em muitos casos, os analistas insistem em utilizar somente a técnica sem uma preparação anterior e em outras situações a mesma técnica é utilizada para elicitar requisitos de todos projetos de uma empresa [Hickey e Davis, 2002]. Além disso, pouca pesquisa tem sido focada na identificação das técnicas de elicitação mais adequadas [Dieste, Lopez e Ramos, 2008].

Considerando a elicitação de requisitos fase crítica no desenvolvimento de software, este trabalho propõe um processo de elicitação de requisitos com foco na escolha da técnica mais adequada para elicitação dependendo do projeto. Um processo para elicitação de requisitos que contemple um conjunto de atividades que permita uma maior interação entre analistas e usuários e, além disso, auxilie na seleção da técnica a ser utilizada, poderá contribuir para que os requisitos levantados correspondam às reais necessidades dos usuários. O processo de elicitação proposto foca na escolha da técnica de elicitação com base nas características do projeto e na colaboração entre envolvidos.

Este trabalho está organizado da seguinte maneira: a Seção 2 descreve os conceitos relacionados à Engenharia de Requisitos e Técnicas de Elicitação. A Seção 3 apresenta os trabalhos relacionados. O processo proposto é descrito na Seção 4. A Seção 5 inclui a descrição dos resultados obtidos enquanto a Seção 6 conclui o trabalho.

\section{Elicitação de Requisitos}

A elicitação corresponde ao processo de levantamento de requisitos. Nesta fase, são levantados os objetivos principais do sistema, identificando assim os problemas 
apresentados pelos usuários e/ou clientes. Em um segundo momento, são identificadas as possíveis soluções para os problemas e objetivos detectados [Lauesen, 2002].

Durante o processo de elicitação, é necessário manter um contato com os usuários e clientes do sistema para entender suas reais necessidades obtendo assim os requisitos do software [Pfleeger, 2004]. Os problemas de elicitação de requisitos não podem ser solucionados apenas com uma abordagem tecnológica, uma vez que o conjunto formado por aspectos sociais, pessoas, informações e contextos assumem importância significativa na elicitação [Belgamo e Martins, 2000].

Na tentativa de minimizar os problemas encontrados na atividade de elicitação de requisitos, existem algumas técnicas que facilitam a comunicação entre clientes e analistas. Estas técnicas são conhecidas como técnicas de elicitação de requisitos.

\subsection{Técnicas de Elicitação de Requisitos}

As técnicas de elicitação surgiram para auxiliar na identificação dos requisitos junto aos usuários. Uma técnica deve explorar características específicas do problema sendo tratado no desenvolvimento de um sistema. Como as características dos problemas variam, é necessário um repertório de métodos para cada classe de problemas [Belgamo e Martins, 2000]. Há muitas técnicas de elicitação publicadas na literatura [Dieste, Lopez e Ramos, 2008]. Entretanto, informações são ainda capturadas, em muitos casos, utilizando somente entrevistas, apesar de haver clara evidência de que entrevistas tradicionais nem sempre são a melhor forma de se extrair requisitos [Carrizo, Dieste e Juristo, 2008]. Nesta seção, apresentamos brevemente algumas técnicas de elicitação.

As Oficinas de Requisitos são reuniões com participação dos desenvolvedores, usuários e outros interessados para definição de requisitos de um sistema em conjunto. A técnica JAD (Joint Application Development) é um exemplo de oficina. JAD visa reunir autoridades representativas e gerenciais em um workshop organizado para promover decisões [Belgamo e Martins, 2000]. Sua aplicação é recomendada quando a necessidade de consenso entre os usuários do sistema se torna fator importante para o desenvolvimento do software. O objetivo dessa técnica é garantir que os usuários se mantenham comprometidos com o levantamento dos requisitos do sistema.

A Prototipagem é uma técnica utilizada com frequiência na elicitação de requisitos. Existem dois tipos de protótipos: os descartáveis e os evolutivos. Protótipos descartáveis são criados com a função de ilustrar para os usuários e/ou clientes do sistema o que o analista entendeu sobre os requisitos que deverão ser contemplados no produto. Essa prototipagem deve ser feita rapidamente e ser concluída em no máximo alguns dias. Protótipos evolutivos são reaproveitados durante a construção do sistema.

A Entrevista é uma técnica que ajuda na captura de conhecimento sobre o domínio do problema, porém, para identificar a solução desses problemas, muitas vezes, se torna necessário utilizar outras técnicas. [Lauesen, 2002]

O uso de Questionários é muito utilizado quando os analistas identificam a necessidade de coletar informações de muitos usuários ao mesmo tempo [Lauesen, 2002]. Quando aplicado, cada usuário responde o questionário individualmente e posteriormente os requisitos são identificados através de análise de respostas fornecidas.

Em sessões de Brainstorming, um grupo de pessoas é reunido, um cenário simulado e um assunto discutido para elicitar os requisitos. As pessoas participantes 
devem se sentir confortáveis o bastante para discutir o assunto sem se sentirem intimidadas. Nenhuma idéia é descartada. Todas idéias são boas idéias [Lauesen, 2002].

\section{Trabalhos Relacionados}

Em [Belgamo e Martins, 2000], é realizada uma análise que visa estudar, comparar e apresentar diferenças e semelhanças entre algumas técnicas de elicitação selecionadas para enfrentar os problemas de elicitação de requisitos. Os autores propuseram um conjunto de parâmetros para avaliação e classificação de algumas técnicas estudadas.

Outro estudo relacionado ao tema foi realizado por [Batista e Carvalho, 2003]. Os autores apresentaram alguns parâmetros relacionados aos projetos e às técnicas que devem ser analisadas no momento da escolha da técnica de elicitação.

No trabalho proposto por [Freitas, Borges e Araújo, 2007], é descrito um processo colaborativo para elicitação de requisitos. Os autores apresentam um processo e uma ferramenta de apoio à colaboração e à negociação na fase de levantamento e definição dos requisitos do sistema. Por meio de um estudo de caso, os autores concluíram que a proposta contribuiu para o desempenho da atividade e aumentou as iniciativas de comunicação entre os stakeholders. Como resultado, a atividade de elicitação ganhou características mais ativas e participativas.

O artigo [Dieste, Lopez e Ramos, 2008] apresenta algumas recomendações sobre as situações em que cada técnica é útil. Estas recomendações são baseadas em uma revisão sistemática de vários estudos empíricos da literatura.

O trabalho [Carristo, Dieste e Juristo, 2008] propõe um framework para apoiar a tomada de decisão quanto à melhor técnica de elicitação de acordo com as características do projeto em questão. O framework define como cada técnica responde a distintas características de projetos.

Este trabalho se diferencia dos citados, pois define um processo de elicitação de requisitos com suporte à seleção da técnica de elicitação de requisitos, bem como permite integração entre os envolvidos. Este processo é apresentado na próxima seção.

\section{O Processo de Elicitação de Requisitos Proposto}

O processo de elicitação proposto conta com os seguintes papéis:

- Analista de Requisitos: Responsável pela seleção da técnica e pela condução da elicitação de requisitos;

- Gerente de Projetos: Responsável por prover informações sobre o projeto para o Analista de Requisitos e também aprovar a técnica indicada pelo processo;

- Cliente/Usuário: Responsável por prover informações para identificação dos requisitos.

O processo baseado na Seleção da Técnica de Elicitação proposto é composto por cinco atividades, conforme ilustrado na Figura 1. Suas atividades são descritas a seguir. 


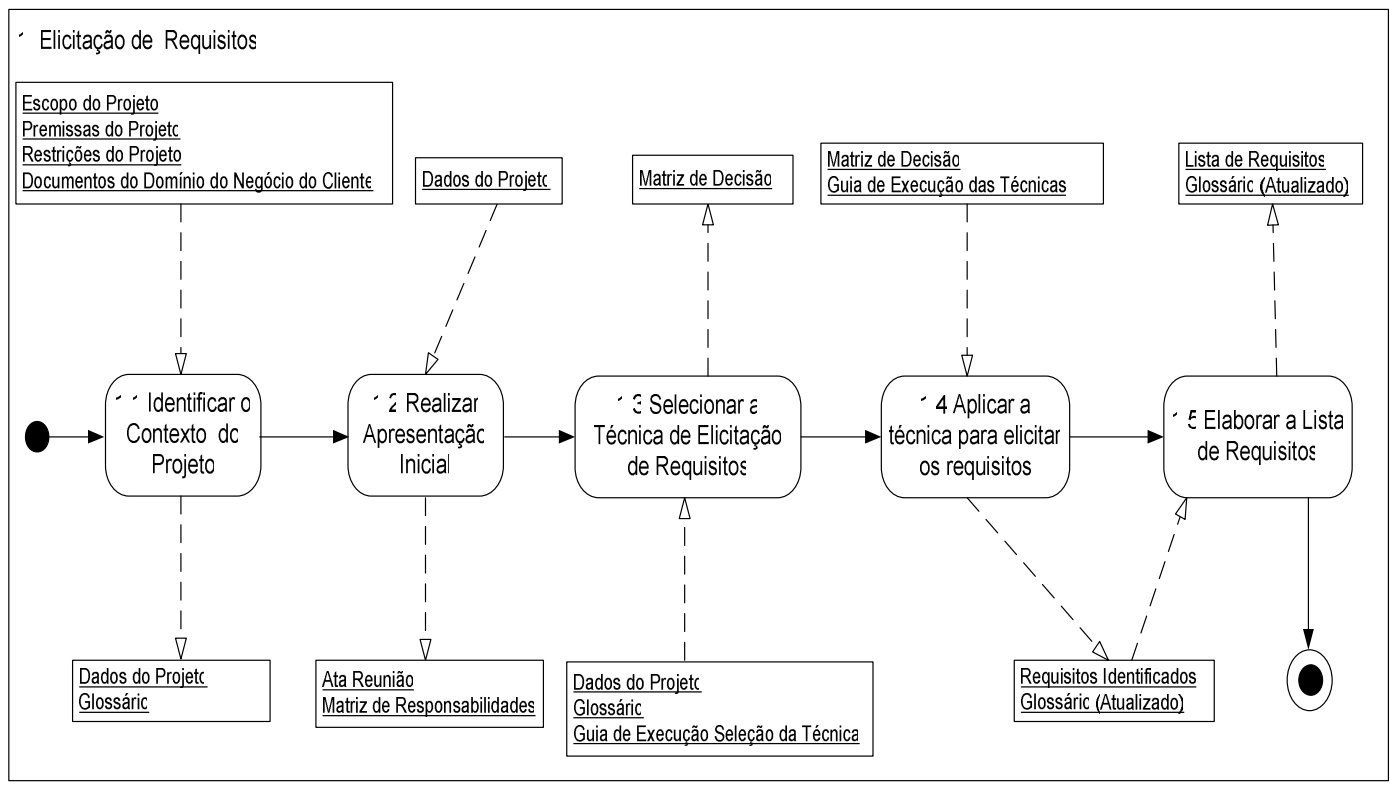

Figura 1. O Processo de Elicitação de Requisitos proposto

\subsection{Atividade: Identificar o Contexto do Projeto}

Esta atividade tem como propósito contextualizar o Analista de Requisitos sobre o Projeto que será desenvolvido. Através de uma reunião com o Gerente do Projeto, o Analista obtém informações relacionadas ao escopo, premissas, restrições e informações relacionadas ao domínio do negócio do cliente antes de realizar um primeiro contato direto com o(s) usuário(s) do sistema. Baseado nas informações obtidas nesta atividade, o Analista de Requisitos poderá analisar o domínio de negócio do cliente e os dados do projeto de uma forma mais contextualizada para prosseguir no processo de elicitação.

Como resultado da atividade, é elaborado um Glossário pelo Analista, que poderá ser atualizado durante a execução do processo de elicitação. Neste Glossário, são registrados os termos relevantes relacionados ao domínio de negócio do cliente visando facilitar a compreensão e comunicação entre os envolvidos no processo de elicitação.

\subsection{Atividade: Realizar Apresentação Inicial}

Esta atividade tem como propósito realizar uma reunião para conscientizar os envolvidos sobre a importância da colaboração do(s) usuário(s) dentro do processo de elicitação de requisitos, uma vez que esta colaboração estará associada ao sucesso do resultado final do sistema que será desenvolvido.

Durante a reunião, o Analista de Requisitos também coleta informações sobre os papéis e responsabilidades dos envolvidos no processo de elicitação. Estas informações serão estruturadas em uma Matriz de Responsabilidades. A Matriz de Responsabilidades permite ao Analista de Requisitos identificar o perfil de cada um dos usuários envolvidos, facilitando assim a condução da elicitação. Uma Ata de Reunião é gerada para documentar os temas abordados e repassá-los a todos os envolvidos. 


\subsection{Atividade: Selecionar a Técnica de Elicitação de Requisitos}

O propósito desta atividade é auxiliar o Analista de Requisitos a escolher uma técnica de elicitação que melhor se adapte às características do projeto, da organização e da equipe de desenvolvimento. Esta atividade foca em orientar o Analista de Requisitos na escolha da técnica a ser utilizada com base em parâmetros relacionados ao projeto.

O processo descrito não restringe o número de técnicas de elicitação que podem ser analisadas para seleção, entretanto, para realizar nossos experimentos, definimos que o processo seria baseado em um universo de 5 técnicas. Procuramos escolher técnicas que são aplicadas para um único usuário e outras que necessitam de um grupo de usuários para serem executadas. Como resultado, as seguintes técnicas foram escolhidas: Brainstorming, Entrevistas, JAD, Questionários e Prototipação Descartável.

[Belgamo e Martins, 2000] apresentam um estudo no qual um conjunto de técnicas, incluindo as selecionadas para compor este processo, são avaliadas de acordo com um grupo de parâmetros. Os autores [Batista e Carvalho, 2003], guiados pelo trabalho de [Belgamo e Martins, 2000], identificaram parâmetros que estão diretamente relacionados aos projetos de software e classificaram as técnicas de acordo com esses.

Um exemplo de parâmetro escolhido é a "Fonte de obtenção dos requisitos". Este foi considerado uma restrição para a seleção, pois há técnicas que focam na elicitação voltada para grupo de usuários e outras aplicadas individualmente.

Dentre os parâmetros apresentados pelos autores, selecionamos os que geralmente impactam nos projetos independente da estrutura da Organização. Os parâmetros selecionados e suas definições segundo os autores são apresentados a seguir:

- Fonte de obtenção dos requisitos: Indica se a técnica é aplicada em grupo ou individualmente. Considerado uma restrição no processo de tomada de decisão;

- Qualidade: Indica se a técnica permite um aprendizado mútuo, resolução de conflito e identificação dos requisitos;

- Tempo: Indica o tempo despendido para a elicitação de requisitos;

- Custo: Indica o custo (relacionado a pessoas e capital) necessário;

- Confiabilidade: Indica se as informações coletadas são confiáveis para o desenvolvimento do projeto;

- Contexto: Indica se a técnica leva em conta o ambiente onde está se realizando a elicitação, ou seja, se a técnica permite que o Analista de Requisitos considere efetivamente o domínio do negócio sobre o qual o sistema será desenvolvido;

- Validação dos requisitos: Indica se a técnica permite validação dos requisitos;

- Necessidade de treinamento: Indica o nível de necessidade de treinamento que o Analista de Requisitos precisará para conhecer e aplicar a técnica na elicitação.

Com o conjunto de técnicas e parâmetros definidos, deve então ser aplicada a tomada de decisão para escolha da técnica de elicitação. [Robbins, 2005] identifica que, de forma geral, o modelo de tomada de decisão racional consiste em: definir o problema; identificar os critérios para a decisão; atribuir pesos específicos para cada um dos critérios definidos; desenvolver alternativas para resolver o problema de forma 
satisfatória e escolher a melhor alternativa, ou seja, o tomador de decisão deve analisar criticamente e avaliar cada uma com base nos critérios estabelecidos como relevantes.

Depois de realizada uma análise das técnicas de tomada de decisão existentes, e considerando o contexto em que se aplica o processo de elicitação proposto neste trabalho, a técnica utilizada para auxiliar o Analista na seleção da técnica de elicitação foi a Matriz de Decisão. Esta técnica consiste em ponderar informações que levarão a possíveis soluções de interesses em linhas e colunas de uma matriz. Para cada intercessão de linha e coluna é aplicada uma pontuação e a matriz pode ainda conter pesos para alguns ou todos os elementos cujos valores precisam ser ressaltados. Normalmente, a análise do resultado na matriz de decisão é feita considerando que o maior valor ponderado constituirá, possivelmente, a melhor decisão a ser tomada.

A Matriz de Decisão é construída estruturando seus dados em linhas e colunas:

- Linhas: Representam cada um dos parâmetros relacionados a projetos de software que são levados em consideração no momento da escolha da técnica;

- Colunas: Representam as técnicas de elicitação. Para cada técnica foram atribuídos pesos em relação a cada um dos parâmetros listados.

Para cada técnica de elicitação, foi atribuído um peso em relação aos parâmetros selecionados, indicando o grau que aquela técnica atende ao parâmetro em questão. Os pesos são valores que correspondem às classificações propostas pelos autores dos trabalhos usados como base. Nestes, cada técnica recebeu classificações de "Baixo" a "Alto" para cada parâmetro avaliado. Neste trabalho, as classificações foram mapeadas para valores de 1 a 3 para calcular o resultado da Matriz. Como o objetivo é atribuir a maior pontuação à técnica mais adequada, os valores foram assinalados aos parâmetros dependendo do objetivo desejado - minimizar ou maximizar o efeito do parâmetro. Assim, parâmetros que devem ser maximizados, como Qualidade, receberam pesos de 1 a 3, para classificações de Baixo a Alto. Parâmetros que devem ser minimizados, como Tempo, receberam pesos de 1 a 3, representando a escala de Alto a Baixo.

Na Figura 2, é possível demonstrar, por exemplo, que as técnicas Brainstorming, JAD e Prototipação Descartável propõem uma metodologia que visa coletar requisitos com alta qualidade. A técnica Questionário, embora apresente um tempo de execução médio em relação à JAD não permite que o contexto do projeto, como informações sobre o domínio de negócio, possa ser explorado no momento da elicitação de acordo com a classificação de [Belgamo e Martins, 2000] e [Batista e Carvalho, 2003].

O Analista define quais parâmetros devem ser priorizados na escolha da técnica que será utilizada para elicitação dos requisitos de acordo com características do projeto (levantadas nas atividades anteriores). Esta definição é realizada através da atribuição do grau de relevância (nota) para cada um dos parâmetros. Este peso pode variar de $0 \mathrm{a}$ 5 pontos. Os valores entre 0 e 2 indicam uma relevância baixa do parâmetro para o projeto. Os valores entre 3 e 5 indicam que o parâmetro apresenta alta relevância. $\mathrm{O}$ Analista deve registrar uma justificativa para cada nota para análises futuras.

Os resultados da matriz são gerados através do somatório das pontuações intermediárias obtidas para cada técnica. Estas pontuações são calculadas através da multiplicação do peso pela nota, atribuída pelo Analista, para cada técnica em relação a cada parâmetro. A técnica mais adequada será a que obtiver a maior pontuação total. 


\begin{tabular}{|c|c|c|c|c|c|c|c|c|c|c|c|c|}
\hline \multicolumn{13}{|c|}{ Matriz de Decisão } \\
\hline Parâmetros & \multicolumn{2}{|c|}{$\begin{array}{c}\text { Colunas de } \\
\text { Preenchimento do } \\
\text { Analista de } \\
\text { Requisitos }\end{array}$} & \multicolumn{2}{|c|}{ Entrevista } & \multicolumn{2}{|c|}{ Questionário } & \multicolumn{2}{|c|}{$\begin{array}{l}\text { Prototipação } \\
\text { Descartável }\end{array}$} & \multicolumn{2}{|c|}{ JAD } & \multicolumn{2}{|c|}{ Brainstorming } \\
\hline $\begin{array}{l}\text { Restriigáo: } \\
\text { Selecione a Fonte } \\
\text { de obtenção dos }\end{array}$ & \multicolumn{2}{|r|}{ Grupo } & \multicolumn{2}{|c|}{ Indivíduo } & \multicolumn{2}{|c|}{ Indivíduo } & \multicolumn{2}{|c|}{ Grupo / Indivíduo } & \multicolumn{2}{|c|}{ Grupo } & \multicolumn{2}{|c|}{ Grupo } \\
\hline & $\begin{array}{l}\text { Sua } \\
\text { Nota }\end{array}$ & $\begin{array}{c}\text { Justificativa } \\
\text { da Nota }\end{array}$ & Peso & Pontuação & Peso & Pontuação & Peso & Pontuação & Peso & Pontuação & Peso & Pontuação \\
\hline Qualidade & 5 & 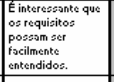 & 2 & 0 & 1 & 0 & 3 & 15 & 3 & 15 & 3 & 15 \\
\hline Tempo Gasto & 5 & $\left|\begin{array}{l}\text { Exiztc urgâncis na } \\
\text { entrega do oiztema. }\end{array}\right|$ & 2 & 0 & 2 & 0 & 2 & 10 & 1 & 5 & 2 & 10 \\
\hline Custo & 0 & 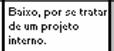 & 2 & 0 & 2 & 0 & 1 & 0 & 1 & 0 & 2 & 0 \\
\hline Confiabilidade & 5 & 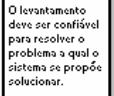 & 3 & 0 & 2 & 0 & 3 & 15 & 3 & 15 & 2 & 10 \\
\hline Contexto & 3 & \begin{tabular}{|l|}
$\begin{array}{l}0 \text { dominio o do } \\
\text { contexto ód d } \\
\text { conhecimento gersal }\end{array}$ \\
\end{tabular} & 3 & 0 & 1 & 0 & 3 & 9 & 3 & 9 & 3 & 9 \\
\hline $\begin{array}{l}\text { Validar } \\
\text { requisitos }\end{array}$ & 3 & 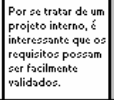 & 3 & 0 & 0 & 0 & 3 & 9 & 3 & 9 & 0 & 0 \\
\hline $\begin{array}{l}\text { Esforço } \\
\text { despendido } \\
\text { com } \\
\text { treinamento }\end{array}$ & 5 & 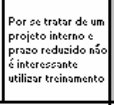 & 2 & 0 & 1 & 0 & 1 & 5 & 1 & 5 & 2 & 10 \\
\hline & & SULTADOS & $\begin{array}{l}\text { Pontuaçăo } \\
\text { Total }\end{array}$ & 0 & $\begin{array}{c}\begin{array}{c}\text { Pontuação } \\
\text { Total }\end{array} \\
\end{array}$ & 0 & $\begin{array}{c}\begin{array}{c}\text { Pontuação } \\
\text { Total }\end{array} \\
\end{array}$ & 63 & $\begin{array}{c}\begin{array}{c}\text { Pontuação } \\
\text { Total }\end{array} \\
\end{array}$ & 58 & $\begin{array}{c}\text { Pontuação } \\
\text { Total }\end{array}$ & 54 \\
\hline
\end{tabular}

Figura 2. Exemplo de matriz de decisão preenchida

\subsubsection{Descrição do Processo de Seleção da Técnica}

Antes de levantar os requisitos do sistema, o Analista de Requisitos deverá selecionar uma técnica que o auxilie na elicitação. Considerando que alguns parâmetros relacionados ao projeto influenciam a escolha da técnica que deverá ser utilizada, o Analista de Requisitos deverá utilizar a Matriz de Decisão, proposta neste processo, para auxiliar a tomada de decisão.

Para guiar o Analista de Requisitos, foi elaborado um Guia de Execução para Seleção da Técnica. Este guia contempla um conjunto de definições, procedimentos e orientações que auxiliam o Analista na execução de cada técnica de elicitação.

Ao preencher a Matriz de Decisão, o Analista de Requisitos deve informar uma nota para cada um dos parâmetros do processo, refletindo assim a importância ou a existência de restrições em relação a este parâmetro no projeto. Por exemplo, para o parâmetro "Qualidade", o Analista de Requisitos deverá se perguntar: "Em que grau o projeto demanda uma técnica que enfoque na qualidade dos requisitos elicitados?". O Analista deverá atribuir uma nota de 0 (baixo) a 5 (alto). Para todos os parâmetros do processo, o Analista de Requisitos deve informar uma nota. Ao final, todas as técnicas que atendem à restrição do processo terão suas respectivas pontuações totais calculadas.

A técnica que obtiver a maior pontuação total na Matriz de Decisão será a indicada pelo Processo de Seleção para que o Analista de Requisitos aplique no Projeto. Entretanto, o processo de seleção atua como uma forma de orientação na escolha da técnica. O Analista de Requisitos poderá escolher uma técnica diferente da que obteve a maior pontuação final, devido à proximidade dos resultados ou por outro motivo justificável. A escolha do Analista deverá ser aprovada pelo Gerente de Projeto.

O Analista de Requisitos deverá registrar a técnica indicada pelo processo, a técnica que ele optou por utilizar, a justificativa da escolha e a técnica que realmente 
será utilizada com aprovação do Gerente de Projeto para a elicitação. As informações geradas durante a execução do processo deverão ser armazenadas para cada projeto. Estas podem compor uma base histórica auxiliando projetos futuros.

É importante ressaltar que o peso de cada parâmetro pode variar de acordo com o projeto em que a técnica é aplicada. Assim, o mesmo Analista de Requisitos poderá atribuir um grau de relevância distinto para o mesmo parâmetro em projetos diferentes.

\subsection{Atividade: Aplicar a Técnica para elicitar os requisitos}

Esta atividade tem como propósito coletar e listar os requisitos para desenvolvimento do sistema. O Analista de Requisitos utilizando a técnica selecionada coleta os requisitos necessários para desenvolvimento do sistema junto ao(s) usuário(s).

Como resultado desta atividade, é gerado um documento, denominado neste processo como Requisitos Identificados, contendo os requisitos elicitados durante a aplicação da técnica. O Glossário também pode ser atualizado caso o Analista de Requisitos identifique alguma necessidade.

\subsection{Atividade: Elaborar a Lista de Requisitos}

Nesta atividade, o Analista de Requisitos elabora a Lista de Requisitos, baseado nos Requisitos Identificados durante a elicitação aplicando a técnica selecionada.

A Lista de Requisitos deve conter uma descrição do sistema em desenvolvimento, a descrição dos requisitos e o critério de aceitação, que consiste nas condições necessárias para verificar se o requisito foi implementado.

Depois de finalizada a Lista de Requisitos, esta deve ser revisada, aprovada e assinada pelo Gerente do Projeto e pelo representante do cliente ou dos usuários. Esta aprovação é intermediária. A atividade formal de verificação/validação dos requisitos não é contemplada neste processo, porém para que se possa dar prosseguimento nas demais atividades de Definição de Requisitos, que não estão incluídas neste processo, é necessária uma validação intermediária do Gerente e do Cliente.

\section{Experimentos e Resultados}

O objetivo deste trabalho foi propor um processo para elicitação de requisitos que auxiliasse o Analista de Requisitos na escolha da técnica de elicitação a ser utilizada bem como provesse uma maior colaboração entre os envolvidos no processo.

A proposta para validação do mesmo consistiu em aplicá-lo em um projeto piloto e posteriormente em estudos de caso de projetos reais. O objetivo do projeto piloto foi verificar que a descrição das atividades poderia ser facilmente seguida e que os artefatos gerados eram suficientes e necessários para o processo de elicitação.

Uma planilha para contabilização do tempo e custo despendidos e uma Checklist de Verificação para validação da Lista de Requisitos foram elaboradas para registrar os dados referentes aos estudos de caso. Estes documentos não fazem parte do processo e foram utilizados apenas com o propósito de realizar a validação dos experimentos.

Nas próximas seções, é descrito em maior detalhe como a verificação foi realizada, os estudos de caso e os resultados alcançados. O objetivo dos estudos de caso apresentados foi comparar uma elicitação realizada com o processo proposto com outra 
realizada com uma metodologia distinta de forma a identificar qual proporcionou melhor atendimento aos objetivos do Cliente.

\subsection{Descrição da Verificação dos Requisitos}

Para validar o processo proposto neste trabalho, dados referentes a tempo e custo das elicitações foram coletados. Além disso, o cliente verificou os requisitos levantados em ambas as elicitações (com e sem o processo proposto) por meio da Checklist de Verificação. Esta checklist contempla itens de verificação que medem o grau de completeza, precisão, consistência e clareza de cada requisito identificado e também da Lista de Requisitos como um todo. Desta forma foi possível atestar o grau de conformidade dos requisitos elicitados em relação ao Contexto das informações e a Qualidade dos requisitos.

Para medir se os parâmetros priorizados pelo Analista de Requisitos, antes da elicitação, foram mais bem atendidos com o processo proposto, cada item da Checklist de Verificação foi relacionado a um parâmetro analisado. Esse relacionamento indica que o item de verificação, se atendido correta e completamente, leva ao atendimento do parâmetro correspondente. A classificação dos itens da Checklist foi baseada na definição de [Belgamo e Martins, 2000] [Batista e Carvalho, 2003].

A Checklist de Verificação é uma planilha na qual os requisitos identificados são dispostos em linhas enquanto os itens de verificação são dispostos nas colunas. $\mathrm{Na}$ intersecção de uma linha e uma coluna, o cliente deve informar se o item em questão está conforme, parcialmente conforme, não conforme ou se ele não sabe avaliar. Para medir de forma simples a verificação dos requisitos, a cada uma das possíveis respostas foram atribuídos valores. Itens conformes receberam valor igual a 1; parcialmente conformes receberam valor igual a 0,5 e itens não conformes valor igual a 0 .

Para melhor comparar os resultados dos estudos de caso, definimos Universo de Requisitos como o número total de requisitos únicos identificados em ambas elicitações (com e sem o processo), ou seja, todos os requisitos do sistema identificados pelo Cliente. Esse número é definido de acordo com a verificação realizada pelo Cliente.

\subsection{Estudo de caso 01 - Sistema de Solicitação de Viagens}

Este estudo de caso foi realizado em uma empresa de Belo Horizonte que comercializa produtos, soluções e serviços diferenciados no segmento de Tecnologia da Informação. A empresa possui processo de desenvolvimento próprio, porém utiliza sempre a técnica de Entrevista para elicitação dos requisitos.

O projeto escolhido consiste em desenvolver um módulo de solicitação de viagens para a empresa. Este módulo deve permitir o gerenciamento do fluxo da solicitação de viagens e manter histórico destas para consultas futuras e relatórios.

Dois Analistas, com perfis similares, levantaram os requisitos deste sistema. Um Analista, que está se especializando em Engenharia de Software, realizou o levantamento utilizando a metodologia da empresa para elicitação (distinta da proposta no processo). O outro Analista, que também está se especializando em Engenharia de Software, utilizou o processo proposto neste trabalho.

O processo proposto indicou a utilização da técnica de Prototipação Descartável e o Analista a aplicou para elicitar os requisitos. O Universo de Requisitos foi 
identificado pelo cliente como sendo composto por 21 requisitos. A elicitação com o processo proposto levantou 21 requisitos $(100 \%)$ enquanto a que não utilizou o processo identificou apenas $6(29 \%)$. O tempo gasto na elicitação sem o processo proposto foi de 8 horas e para a elicitação com o processo foi de 6 horas.

Através das análises realizadas nos resultados deste estudo, observou-se que na escolha da técnica de elicitação, o Analista que utilizou o processo proposto, priorizou:

- Elicitação em um tempo reduzido devido à urgência da entrega do sistema;

- Coletar o máximo de requisitos com qualidade para que os mesmos possam ser facilmente entendidos;

- Elicitar requisitos confiáveis, uma vez que foi entendido que a solicitação de viagens era um processo delicado da empresa que estava gerando prejuízos;

- Não demandar tempo com treinamento para elicitar os requisitos.

Analisando os resultados apresentados, verificou-se que em relação à elicitação realizada sem o processo proposto, o tempo da elicitação realizada com o processo proposto foi menor. Outro indicador que merece destaque é que a elicitação realizada com o processo proposto apresentou requisitos com maior Qualidade, conforme pode ser verificado na Tabela 1. Este resultado pode ser vinculado ao fato de o Analista de Requisitos explorar melhor o contexto do sistema e o domínio de negócio do cliente.

\subsection{Estudo de caso 02 - Sistema de Comércio Eletrônico}

Este estudo de caso foi realizado em uma empresa de Tecnologia de Informação que está iniciando suas atividades no mercado e visa prestar serviços de $e$-commerce. Esta empresa ainda não possui um processo definido para elicitação de requisitos ou mesmo para outras atividades relacionadas à Definição e Gerência de Requisitos.

O projeto consiste em desenvolver o embrião de um sistema que deverá oferecer serviços de comércio eletrônico para estabelecimentos comerciais provendo um ambiente compartilhado para que esses possam divulgar e vender seus produtos.

Dois Analistas, com perfis similares, levantaram os requisitos. Um Analista, graduando em Sistemas de Informação (SI), realizou o levantamento através de Entrevista com usuário. Ao final, foi gerado documento com requisitos elicitados. $\mathrm{O}$ outro Analista, também graduando em SI, utilizou o processo proposto neste trabalho.

O processo indicou a utilização de Entrevista e o Analista aplicou a mesma para elicitar os requisitos. O tempo gasto na elicitação sem o processo proposto foi de 2 horas e para a elicitação com o processo foi de 2,5 horas. O Universo de Requisitos foi identificado pelo cliente como sendo igual a 23 requisitos. A elicitação com o processo levantou 23 requisitos (100\%) e a que não utilizou identificou 15 (65\%).

Através das análises realizadas nos resultados obtidos, observou-se que na escolha da técnica de elicitação, o Analista que utilizou o processo proposto, priorizou:

- Elicitação em um tempo reduzido devido às restrições do prazo do projeto;

- Elicitar requisitos confiáveis e contextualizados, pois informações com tais características no domínio de negócio do cliente são importantes para o site;

- Não demandar tempo com treinamento para elicitar os requisitos. 
Durante a análise dos resultados, verificou-se a diferença no tempo de execução entre os experimentos foi de apenas 30 minutos e pode ser considerada pequena quando analisamos os demais benefícios relacionados à Qualidade.

Embora em ambas elicitações, a técnica de levantamento de requisitos utilizada tenha sido Entrevista, na elicitação realizada com o processo proposto os requisitos listados apresentaram maior qualidade. Este resultado pode ser vinculado ao fato de o Analista de Requisitos explorar o contexto e domínio de negócio.

\subsection{Estudo de caso 03 - Sistema de Gerenciamento de Erros}

O terceiro estudo de caso foi realizado em outra empresa de desenvolvimento de software de Belo Horizonte. Esta empresa presta consultoria em gerenciamento de projetos e desenvolve ferramentas integradas à solução EPM (Enterprise Project Management) e Sharepoint da Microsoft. A empresa possui metodologia própria para levantamento de requisitos, mas utiliza somente a técnica de Entrevista.

O projeto consiste em desenvolver um sistema simplificado de rastreamento de erros ("Bug Tracking"). Este sistema deverá oferecer funcionalidades para gerenciamento de erros detectados nos projetos desenvolvidos pela empresa.

Dois Analistas, com perfis similares, levantaram os requisitos. Um Analista, graduando em SI, realizou o levantamento utilizando a metodologia da empresa, distinta do processo proposto. Ao final da elicitação, foi gerado documento com os requisitos levantados. O outro Analista, também graduando em SI utilizou o processo proposto.

O processo indicou o uso de Entrevista e o Analista aplicou a mesma para elicitar os requisitos. $O$ tempo gasto para a elicitação sem o processo proposto foi de 1 hora e para a elicitação realizada com o processo foi de 2,5 horas. O Universo de Requisitos foi identificado pelo cliente como sendo de 24 requisitos. A elicitação com o processo levantou 24 requisitos (100\%) e a que não utilizou identificou $11(46 \%)$.

$\mathrm{Na}$ escolha da técnica, o Analista que utilizou o processo proposto, priorizou:

- Elicitar requisitos confiáveis e de qualidade, para não prejudicar o desenvolvimento do sistema;

- Não demandar treinamento para aprender como aplicar a técnica de elicitação de requisitos, uma vez que o Analista de Requisitos está alocado para outro projeto.

Verificou-se que em relação à elicitação realizada sem o processo proposto o tempo da elicitação realizada com o processo proposto foi maior. Porém esta diferença pode ser considerada investimento para melhor levantar os requisitos. De acordo com os critérios do Analista no momento da seleção da técnica, o tempo gasto para a elicitação não era uma restrição e os indicadores apresentaram maior qualidade para os requisitos elicitados durante o levantamento realizado com o processo proposto neste trabalho.

Este resultado pode ser vinculado ao fato do Analista, que aplicou o processo proposto, explorar melhor o contexto no qual o sistema será desenvolvido e estabelecer critérios para selecionar uma técnica para auxiliá-lo no levantamento dos requisitos.

\subsection{Estudo de caso 04 - Sistema de Gerenciamento de Comércio em Bairro}

O quarto estudo de caso foi realizado para a elicitação de requisitos de um sistema cujo objetivo é o gerenciamento de uma pequena loja que comercializa produtos alimentícios 
em um bairro de Belo Horizonte. Este estudo de caso foi realizado pela mesma empresa de Tecnologia de Informação na qual o estudo de caso 02 foi executado. Esta empresa não possui um processo definido.

O sistema irá oferecer funcionalidades para o registro simplificado de vendas, controle do fluxo de caixa, controle de estoque dos produtos e emissão de relatórios.

Para realizar o experimento, foi necessário novamente que dois Analistas, com perfis similares, ambos graduandos em SI, levantassem os requisitos. Um Analista de Requisitos realizou o levantamento através de Entrevista com o usuário. Ao finalizar sua elicitação, foi gerado um documento contendo os requisitos elicitados. O outro Analista realizou o levantamento utilizando o processo proposto neste trabalho.

O processo indicou o uso de Brainstorming e o Analista de Requisitos aplicou a mesma para elicitar os requisitos. O tempo gasto para a elicitação sem a aplicação do processo proposto foi de 1 hora enquanto para a elicitação realizada com o processo foi de 3,5 horas. O Universo de Requisitos foi identificado pela avaliação do cliente como sendo composto por 35 requisitos. A elicitação com o processo proposto levantou 35 requisitos (100\%) enquanto a que não utilizou identificou 11 (31\%).

$\mathrm{Na}$ escolha da técnica, o Analista que utilizou o processo proposto, priorizou:

- Elicitar requisitos de qualidade através da obtenção de informações confiáveis;

- Uma técnica que permitisse que as informações sobre o domínio de negócio do cliente fossem exploradas;

- Não exceder o custo previsto para o levantamento de requisitos.

Verificou-se que em relação à elicitação realizada sem o processo proposto o tempo da elicitação realizada com o processo proposto foi maior. Porém, novamente, esta diferença pode ser considerada investimento para um melhor levantamento dos requisitos. O tempo gasto para a elicitação não era uma restrição e os indicadores apresentaram maior qualidade para os requisitos elicitados durante o levantamento realizado com o processo proposto neste trabalho.

Na próxima seção, os resultados dos experimentos são apresentados.

\subsection{Consolidação dos Resultados}

Os resultados confirmam que os requisitos elicitados com o uso do processo proposto apresentaram maior Qualidade e permitiram identificar melhor o Contexto do projeto na concepção do cliente e a partir dos mesmos foi possível chegar às conclusões seguintes.

A Tabela 1 apresenta os resultados obtidos nos estudos de forma sumarizada.

\section{Conclusões e Trabalhos Futuros}

Neste trabalho foi proposto um processo que auxilia Analistas na escolha da técnica de elicitação, com base em características relevantes do projeto do sistema desenvolvido, e fornece maior colaboração entre os envolvidos nesta fase do desenvolvimento.

Experimentos foram realizados com o objetivo de avaliar o processo proposto em relação a outras metodologias utilizadas em empresas de desenvolvimento de software para a elicitação de requisitos. Através destes, observou-se que os Analistas 
não se preparam de forma adequada para realizar a elicitação e, por isto, em muitos casos, ficam limitados no momento de executar o levantamento com o Cliente.

Tabela 1. Resultados Sumarizados dos Estudos de Caso

\begin{tabular}{|c|c|c|c|c|c|c|c|c|}
\hline $\begin{array}{l}\text { Dados Obtidos/ } \\
\text { Itens Avaliados }\end{array}$ & \multicolumn{2}{|c|}{$\begin{array}{c}\text { Estudo de Caso } 1 \\
\text { Sistema de Viagens }\end{array}$} & \multicolumn{2}{|c|}{$\begin{array}{c}\text { Estudo de Caso } 2 \\
\text { Comércio Eletrônico }\end{array}$} & \multicolumn{2}{|c|}{$\begin{array}{c}\text { Estudo de Caso } 3 \\
\text { Bug Tracking }\end{array}$} & \multicolumn{2}{|c|}{$\begin{array}{c}\text { Estudo de Caso } 4 \\
\text { Comércio em Bairro }\end{array}$} \\
\hline \multirow{2}{*}{$\begin{array}{l}\text { Universo de } \\
\text { Requisitos }\end{array}$} & \multicolumn{2}{|c|}{21} & \multicolumn{2}{|c|}{23} & \multicolumn{2}{|c|}{24} & \multicolumn{2}{|c|}{35} \\
\hline & $\begin{array}{c}\text { Com } \\
\text { Processo }\end{array}$ & $\begin{array}{c}\text { Sem } \\
\text { Processo }\end{array}$ & $\begin{array}{c}\text { Com } \\
\text { Processo }\end{array}$ & $\begin{array}{c}\text { Sem } \\
\text { Processo }\end{array}$ & $\begin{array}{c}\text { Com } \\
\text { Processo }\end{array}$ & $\begin{array}{c}\text { Sem } \\
\text { Processo }\end{array}$ & $\begin{array}{c}\text { Com } \\
\text { Processo }\end{array}$ & $\begin{array}{c}\text { Sem } \\
\text { Processo }\end{array}$ \\
\hline Técnica & Protótipo & Entrevista & Entrevista & Entrevista & Entrevista & Entrevista & Brainstorming & Entrevista \\
\hline Tempo (hrs) & 6 & 8 & 2,5 & 2 & 2,5 & 1 & 3,5 & 1 \\
\hline $\begin{array}{l}\mathbf{N}^{0} \text { Requisitos } \\
\text { Identificados }\end{array}$ & 21 & 6 & 23 & 15 & 24 & 11 & 35 & 11 \\
\hline $\begin{array}{l}\text { Porcentagem } \\
\text { (Universo)* }\end{array}$ & $100 \%$ & $29 \%$ & $100 \%$ & $65 \%$ & $100 \%$ & $46 \%$ & $100 \%$ & $31 \%$ \\
\hline Qualidade*** & $98 \%$ & $100 \%$ & $100 \%$ & $74 \%$ & $98 \%$ & $75 \%$ & $95 \%$ & $51 \%$ \\
\hline $\begin{array}{c}\text { Qualidade } \\
\text { Ponderada**** }\end{array}$ & $98 \%$ & $29 \%$ & $100 \%$ & $48 \%$ & $98 \%$ & $34 \%$ & $95 \%$ & $16 \%$ \\
\hline Contexto***** & $100 \%$ & $50 \%$ & $100 \%$ & $50 \%$ & $100 \%$ & $25 \%$ & $100 \%$ & $25 \%$ \\
\hline$*$ & \multicolumn{8}{|c|}{$\begin{array}{l}\text { Representa a porcentagem do número de requisitos levantados, em cada elicitação (com e sem o processo), em relação } \\
\text { ao número de requisitos total identificados pelo cliente (Universo de Requisitos). Indica o quão completa está a lista } \\
\text { de requisitos em relação ao Universo de Requisitos, para cada estudo de caso. }\end{array}$} \\
\hline$* *$ & \multicolumn{8}{|c|}{$\begin{array}{l}\text { Representa o grau de conformidade dos requisitos, de acordo com avaliação do cliente, em cada elicitação } \\
\text { isoladamente (com e sem o processo), em relação aos itens da Checklist de Verificação relacionados à Qualidade. }\end{array}$} \\
\hline$* * *$ & \multicolumn{8}{|c|}{$\begin{array}{l}\text { Representa o grau de conformidade dos requisitos identificados em cada elicitação, de acordo com a avaliação do } \\
\text { cliente, em relação aos itens da Checklist de Verificação relacionados à Qualidade ponderados pelo Universo de } \\
\text { Requisitos. Este indicador permite a comparação dos resultados das elicitações (com e sem o processo) em cada } \\
\text { estudo de caso, relacionados à Qualidade dos requisitos identificados, dentro de um universo único de requisitos. }\end{array}$} \\
\hline$* * * *$ & \multicolumn{8}{|c|}{$\begin{array}{l}\text { Representa o grau de conformidade dos requisitos identificados, de acordo com avaliação do cliente, em cada } \\
\text { elicitação em relação aos itens da Checklist de Verificação relacionados ao Contexto. Indica o quão dentro do } \\
\text { contexto do domínio de negócio os requisitos identificados nos estudos de caso (com e sem o processo) estão. }\end{array}$} \\
\hline
\end{tabular}

Esta observação pode ser justificada também pelos resultados obtidos relacionados à Qualidade e Contexto. De maneira geral, os requisitos elicitados seguindo o processo desenvolvido neste trabalho apresentaram resultados melhores em relação às elicitações realizadas com outras metodologias. Isso se deve aos fatoreschave priorizados pelo processo. O primeiro relacionado aos critérios para selecionar a técnica de elicitação mais adequada para o levantamento dos requisitos e o segundo por permitir maior colaboração e interatividade entre Analistas, Gerentes e Clientes.

Nos casos em que o processo proposto foi aplicado, as técnicas indicadas atenderam aos critérios estabelecidos pelos Analistas na tomada de decisão e os resultados das elicitações contemplaram requisitos que correspondiam às necessidades do Cliente, de acordo com as verificações realizadas pelo mesmo. Isto indica que o entendimento do contexto do projeto, o estabelecimento de critérios para escolha da técnica e a interatividade entre os envolvidos contribuem para um levantamento de requisitos mais próximo das reais necessidades.

É importante ressaltar que os estudos de caso não acompanharam o desenvolvimento dos requisitos. Entretanto, análises dos experimentos permitem inferir que o processo de elicitação focado na seleção da técnica poderá ser aplicado com 
sucesso. Vale destacar que o perfil do Analista que conduz a elicitação pode influenciar os resultados, porém, houve o cuidado de selecionar Analistas com perfis próximos.

Pretende-se realizar experimentos para acompanhar a maturidade dos requisitos até o fim do desenvolvimento, após aplicação do processo. Assim, poder-se-á observar se os requisitos provaram ter os atributos avaliados pelo Cliente ao final da elicitação.

Foi observado que pode haver uma diferenciação ainda maior entre as técnicas em relação aos parâmetros pelos quais as mesmas foram classificadas. Nos trabalhos utilizados como base para definição deste processo [Belgamo e Martins, 2000] [Batista e Carvalho, 2003], há parâmetros com o mesmo peso para várias técnicas. As diferenças entre as técnicas podem ser mais bem exploradas diferenciando seus pesos ou mesmo novos parâmetros poderão ser incluídos. Trabalhos recentes [Dieste, Lopez e Ramos, 2008] [Carristo, Dieste e Juristo, 2008] apresentam classificações mais detalhadas sobre as técnicas de elicitação que poderiam ser usadas neste sentido.

\section{Referências}

Batista, E. A; Carvalho, A. M. B. R. (2003) "Uma Taxonomia Facetada para Técnicas de Elicitação de Requisitos". WER03 - Workshop em Engenharia de Requisitos.

Belgamo, A. e Martins, L. E. G (2000). "Estudo Comparativo sobre as Técnicas de Elicitação de Requisitos do Software". In: XX Congresso Brasileiro da Sociedade Brasileira de Computação (SBC), Curitiba - Paraná.

Carrizo, D.; Dieste, O. e Juristo, N. (2008) "Study of Elicitation Techniques Adequacy". In Workshop on Engenharia de Requisitos (WER 2008), Barcelona, Espanha.

Dieste, O.; Lopez, M. e Ramos, F. (2008) "Updating Systematic Review about Selection of Software Requirements Elicitation Techniques". In Workshop on Engenharia de Requisitos (WER 2008), Barcelona, Espanha.

Freitas, D. P.; Borges, M. R. S; Araújo, R. M. (2007) "Colaboração e Negociação na Elicitação de Requisitos" In: X Workshop Iberoamericano de Ingeniería de Requisitos y Ambientes de Software (IDEAS 07), Isla de Margarita

Hickey, A. M. e Davis, A. M. (2002) "Requirements Elicitation and Elicitation Technique Selection: A Model for Two Knowledge-Intensive Software Development Processes". In: Proceedings of Hawaii International Conference on System Sciences

Lauesen, S. (2002) "Software Requirements Styles and Techniques". Elicitation. England: A Personal Education Limited, 2002. Cap.8 p.331-372.

Pfleeger, S. L. (2004) "Engenharia de software: teoria e prática". Identificando Requisitos 2a .ed. São Paulo: Prentice Hall. Cap.4 p.111-138.

Robbins, Stephen P. "Comportamento Organizacional. Percepção e Tomada de Decisões Individual". 11ª Ed. São Paulo: Pearson Prentice Hall, 2005. Cap.5, p.103 125.

Standish Group International (1995). "The Chaos Report"; http://net.educause.edu/ir/library/pdf/NCP08083B.pdf. 